\title{
Esquemas disfuncionales tempranos: una medida compleja en estudiantes peruanos
}

\section{Early maladaptive schemas: A complex measure in Peruvian students}

\author{
Anthony Copez-Lonzoy* \\ Escuela Profesional de Psicología, \\ Universidad de San Martín de Porres, Perú
}

\section{Resumen}

Esta investigación tiene como objetivo analizar las propiedades psicométricas del Young Schema Questionarie Short Form - 75 (YSQ-SF) en una muestra de 400 estudiantes universitarios, con edades entre 17 y 57 años $(M=23.3 ; D E=5.36)$. Para analizar la estructura interna se realizó un modelo de análisis factorial exploratorio (AFE) por la complejidad factorial presentada en estudios previos que evidenció ocho factores. Posteriormente, se llevó a cabo el análisis factorial confirmatorio al comparar la solución presentada por el AFE. Se obtuvo un alto nivel de consistencia interna. Se concluye que la (YSQ-SF) presenta adecuadas propiedades psicométricas, que apoyan su uso como instrumento de evaluación de los esquemas disfuncionales tempranos en universitarios.

Palabras clave: esquemas disfuncionales; análisis factorial; modelos complejos

\begin{abstract}
The objective of this paper is to analyze the psychometric properties of the Young Schema Questionnaire - Short Form (YSQ-SF, 75-item version) in a sample of 400 university students between ages 17 and $57(\mathrm{M}=23.3$; $\mathrm{SD}=$ 5.36). To analyze the internal structure, an exploratory factor analysis (EFA)
\end{abstract}

Este es un artículo Open Access bajo la licencia Creative Commons Atribución-NoComercial-Compartirlgual 4.0

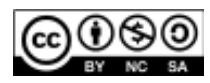


model was used due to the factor complexity found in previous studies, which showed eight factors. Subsequently, a confirmatory factor analysis was carried out by comparing the solution resulting from the EFA. A high level of internal consistency was obtained. It is concluded that the YSQ-SF has adequate psychometric properties, which support its use as an instrument for evaluating early dysfunctional schemas in university students.

Keywords: dysfunctional schemas; factor analysis; complex models

\section{Introducción}

Los patrones desadaptativos generan una estructura compleja que va cimentando recursos disfuncionales en las personas. Estos recursos cognitivos (pensamientos aprendidos desde la infancia) y emocionales (como factores de escape y alivio sobre las situaciones pasadas) son referidos como Esquemas Disfuncionales Tempranos (EDT), que se inician al comienzo del desarrollo humano y van repitiéndose a lo largo de la vida (Young, Klosko, \& Weishmar, 2013). Los EDT se originan en los primeros años de vida por la acumulación de interacciones contraproducentes (tempranas) con personas significativas (diversos componentes familiares o amigos), que pueden ser latentes por mucho tiempo y ser activados ante eventos estresantes (Young \& Klosko, 2001; Young et al., 2013). Cuando son activados por acontecimientos que guardan relación con ellos, en la mayoría de ocasiones, resultan como parte de las distorsiones de pensamiento que suelen ser fuertes respuestas emocionales que generan conductas problemáticas (Young et al., 2013).

Los individuos con esquemas en esta dimensión son incapaces de establecer apegos seguros y satisfactorios con otras personas, creen que sus necesidades de seguridad, protección y amor no serán satisfechas (Young et al., 2013) como un cuerpo estructurado de conocimientos o grupo de creencias que son utilizados para el procesamiento de información, siendo a su vez, representaciones organizadas de la experiencia pasada, estas diferentes experiencias formarían los diversos esquemas (Caro, 2011), desarrollándose la primera gran dimensión de desconexión/rechazo que agrupan los esquemas de abandono/inestabilidad, desconfianza/abuso, privación emocional, imperfección/vergüenza, aislamiento social; la dimensión de autonomía 
deteriorada agrupa los esquemas de dependencia/ incompetencia, vulnerabilidad al daño o a la enfermedad, enmarañamiento y fracaso; la dimensión de límites deteriorados engloba los esquemas de grandiosidad, autocontrol insuficiente/autodisciplina; la dimensión de orientación hacia los demás comprende los esquemas de subyugación y autosacrificio; finalmente, la dimensión de vigilancia excesiva agrupa los esquemas negatividad/ pesimismo y control excesivo/inhibición emocional (Young \& Kolsko, 2007).

El entendimiento de la posible aparición de cada patrón es útil a nivel clínico y de investigación. Contar con instrumentos de medida precisos y confiables es necesario para identificar la posible manifestación de un constructo complejo; por ello, se construyó el Young Schema Questionarie Short Form - 75 (YSQ-SF), instrumento que cuenta con una estructura interna compleja, sin embargo, no se presentan todos los EDT en sus diferentes estudios sobre propiedades psicométricas (Baranoff, Oei, Cho, \& Kwon, 2006; Kriston, Schäfer, von Wolff, Härter, \& Hölzel, 2012; Londoño et al., 2012; Mauchand, Lachenal-Chevallet, \& Cottraux, 2011). Por lo expuesto, el objetivo de este estudio es analizar las propiedades psicométricas, la estructura interna y fiabilidad de las puntuaciones del cuestionario YSQ-SF en estudiantes universitarios.

\section{Método}

\section{Diseño}

Es una investigación de tipo instrumental, estos estudios están encaminados al desarrollo de escalas de medida psicológica. Se incluye, tanto su diseño como adaptación, como el estudio de las propiedades psicométricas (Ato, López, \& Benavente, 2013; Montero \& León, 2002, 2005).

\section{Participantes}

La muestra la conforman 400 estudiantes universitarios con un rango de edad de 17 a 57 años $(M=23.3 ; D E=5.36)$. El 80.3\% de los participantes mujeres; $52.7 \%$ estudiantes y $87.6 \%$ solteros. 


\section{Instrumento}

- Young Schema Questionarie Short Form - 75 (YSQ-SF).

Cuestionario de Esquemas de Young - Forma reducida.

Construido por Young y Brown en 1994 tiene por objetivo evaluar quince esquemas disfuncionales, los cuales están organizados en cinco dominios. El YSQ-SF es un cuestionario de 75 ítems, cada uno de los esquemas está formado por 5 ítems y a las personas participantes se les pide que puntúen los ítems usando una escala de 6 puntos $(1=$ Totalmente falso, $2=\mathrm{La}$ mayoría de las veces falso, 3 = Más falso que verdadero, $4=$ En ocasiones verdadero, $5=$ La mayoría de las veces verdadero, y $6=$ Me describe perfectamente). Numerosos estudios han apoyado la validez de este cuestionario, sugiriendo una alta sensibilidad de la prueba para identificar psicopatología (Londoño et al., 2012).

En cuanto a la estructura del YSQ-SF, en Francia, Lachenal-Chevallet, Mauchand, Cottraux, Bouvard y Martin (2006), mediante análisis factorial exploratorio con 263 participantes, encontraron catorce factores, de los cuales 13 coincidían con los propuestos por Young. Además, hallaron diferencias significativas entre hombres y mujeres en los esquemas de limites insuficientes, privación emocional, desconfianza/maltrato, con puntuaciones superiores en hombres. La primera publicación que incluyó análisis factorial confirmatorio fue realizada por Welburn, Coristine, Dagg, Pontefract y Jordan (como se citó en Londoño et al., 2012), en población clínica, con 196 pacientes. Se obtuvo una buena consistencia interna del YSQ-SF, con un alfa de Cronbach entre .76 y .93 para los 15 componentes. Se identificaron diferencias significativas en las puntuaciones de hombres y mujeres en los esquemas de autosacrificio, apego, fracaso, abandono y defectuosidad, con puntuaciones superiores en mujeres. Ningún esquema de los dominios sobrevigilancia y falta de límite presentó diferencias significativas.

\section{Procedimiento}

De acuerdo con los objetivos de la investigación se efectuó la aplicación del cuestionario a un grupo de universitarios $(n=20)$ para la identificación 
de problemas en la compresión de los ítems. Seguido, se coordinó la intervención de encuestadores con las autoridades del departamento académico, y se informó sobre las implicancias del estudio, señalando la condición voluntaria y anónima de los participantes en la presente investigación. Se aplicó en grupos de 30 o 40 estudiantes, los cuales previamente fueron informados sobre los objetivos del estudio, así como del anonimato y duración (aproximadamente 20 minutos). Cabe reiterar, que solo se contó con los participantes que accedieron de manera voluntaria y no remunerada.

Para evaluar la estructura interna del cuestionario YSQ-SF fue considerado el enfoque de análisis factorial exploratorio de manera inicial; esto se debe a la complejidad entre las diversas estructuras reportadas en estudios anteriores, paso siguiente, se realizó un modelo de análisis factorial confirmatorio (AFC) que tomo en cuenta matrices Pearson debido al escalamiento de los ítem con el método de máxima verosimilitud robusta (Holgado-Tello, Chacón-Moscoso, Barbero-García, \& Vila-Abad, 2010; Lee, Poon, \& Bentler, 1995). Para el análisis del modelo de medida fueron utilizados puntos de corte de los índices de bondad de ajuste (Hu \& Bentler, 1998; Lomax \& Schumager, 2017) $x^{2}$ corregido por Satorra y Bentler (SB$\left.\mathrm{X}^{2}\right)$ como índice global, para la medida incremental del modelo fue utilizado el índice de ajuste comparativo > .90 (CFI), para la medida de la parsimonia se utilizó la raíz del error cuadrático media de aproximación $\leq$ .06 (RMSEA) y adicionalmente la raíz promedio de los residuos estandarizados $\leq .08$ (SRMR). Para evaluar la fiabilidad de las puntuaciones fueron identificados otros modelos confirmatorios para el uso pertinente del coeficiente alfa (Cronbach, 1951; Graham, 2006). Los análisis fueron realizados con el programa $\mathrm{R}$ Studio 3.1.6.

\section{Resultados}

\section{Análisis de ítems}

En la Tabla 1 se muestran las puntuaciones de la media $(M)$, la desviación estándar $(D E)$ que evidencian las puntuaciones media más baja para los ítems 18 «Yo no formo parte de nada; soy un solitario», 21 «Ningún hombre/mujer desearía amarme una vez que viera mis defectos», 22 «Nadie querría estar cerca 
Tabla 1

Análisis descriptivo de los ítems

\begin{tabular}{|c|c|c|c|c|c|c|c|c|c|}
\hline Ítems & $M$ & $D E$ & $g^{1}$ & $g^{2}$ & Items & $M$ & $D E$ & $g^{1}$ & $g^{2}$ \\
\hline CEY_1 & 2.39 & 1.496 & .771 & -.630 & CEY_39 & 1.72 & 1.115 & 1.816 & 3.058 \\
\hline CEY_2 & 2.06 & 1.274 & 1.073 & .132 & CEY_40 & 1.79 & 1.176 & 1.613 & 2.195 \\
\hline CEY_3 & 1.79 & 1.163 & 1.588 & 1.984 & CEY_41 & 2.38 & 1.449 & .859 & -.172 \\
\hline CEY_4 & 2.08 & 1.289 & 1.181 & .568 & CEY_42 & 2.08 & 1.187 & 1.157 & .979 \\
\hline CEY_5 & 2.41 & 1.480 & .864 & -.362 & CEY_43 & 1.82 & 1.142 & 1.682 & 2.682 \\
\hline CEY_6 & 2.14 & 1.359 & 1.248 & .762 & CEY_44 & 1.88 & 1.180 & 1.631 & 2.512 \\
\hline CEY_7 & 1.85 & 1.127 & 1.554 & 2.201 & CEY_45 & 1.67 & 1.103 & 2.135 & 4.690 \\
\hline CEY_8 & 2.14 & 1.275 & 1.197 & .942 & CEY_46 & 1.71 & 1.116 & 2.115 & 4.743 \\
\hline CEY_9 & 2.17 & 1.274 & .997 & .227 & CEY_47 & 1.48 & .882 & 2.206 & 5.084 \\
\hline CEY_10 & 1.90 & 1.233 & 1.579 & 2.013 & $\mathrm{CEY}_{-} 48$ & 1.56 & .936 & 2.051 & 4.463 \\
\hline CEY_11 & 2.12 & 1.269 & 1.131 & .619 & CEY_49 & 1.53 & .892 & 2.172 & 5.504 \\
\hline CEY_12 & 2.00 & 1.202 & 1.338 & 1.345 & CEY_50 & 1.72 & 1.081 & 1.877 & 3.541 \\
\hline CEY_13 & 1.98 & 1.212 & 1.336 & 1.289 & CEY_51 & 2.20 & 1.235 & 1.105 & .861 \\
\hline CEY_14 & 2.50 & 1.382 & .845 & .040 & CEY_52 & 2.57 & 1.415 & .804 & -.039 \\
\hline CEY_15 & 2.36 & 1.397 & .980 & .166 & CEY_53 & 2.19 & 1.341 & 1.220 & .824 \\
\hline CEY_16 & 1.75 & 1.119 & 1.811 & 3.198 & CEY_54 & 2.77 & 1.521 & .671 & -.505 \\
\hline CEY_17 & 2.67 & 1.606 & .694 & -.617 & CEY_55 & 2.27 & 1.362 & 1.157 & .719 \\
\hline CEY_18 & 1.50 & 1.018 & 2.575 & 6.868 & CEY_56 & 2.46 & 1.480 & .815 & -.335 \\
\hline CEY_19 & 1.51 & .980 & 2.398 & 6.146 & CEY_57 & 2.30 & 1.451 & 1.124 & .412 \\
\hline CEY_20 & 1.57 & 1.000 & 2.212 & 4.988 & CEY_58 & 2.07 & 1.339 & 1.360 & 1.181 \\
\hline CEY_21 & 1.50 & 1.000 & 2.766 & 8.296 & CEY_59 & 2.36 & 1.503 & 1.056 & .183 \\
\hline CEY_22 & 1.38 & .876 & 3.017 & 9.819 & CEY_60 & 2.07 & 1.367 & 1.343 & 1.049 \\
\hline CEY_23 & 1.23 & .616 & 3.483 & 13.912 & CEY_61 & 2.63 & 1.483 & .681 & -.460 \\
\hline CEY_24 & 1.50 & 1.026 & 2.405 & 5.628 & CEY_62 & 3.37 & 1.609 & .025 & -1.089 \\
\hline CEY_25 & 1.43 & .849 & 2.640 & 8.173 & CEY_63 & 4.04 & 1.560 & -.496 & -.749 \\
\hline CEY_26 & 1.74 & 1.114 & 1.913 & 3.682 & CEY_64 & 3.12 & 1.521 & .199 & -.917 \\
\hline CEY_27 & 1.55 & 1.014 & 2.341 & 5.744 & CEY_65 & 2.24 & 1.361 & 1.101 & .549 \\
\hline CEY_28 & 1.63 & .946 & 2.023 & 4.812 & CEY_66 & 2.31 & 1.364 & 1.055 & .403 \\
\hline CEY_29 & 1.54 & .942 & 2.138 & 4.900 & CEY_67 & 2.71 & 1.523 & .668 & -.564 \\
\hline CEY_30 & 1.52 & .929 & 2.302 & 6.008 & CEY_68 & 3.10 & 1.683 & .318 & -1.135 \\
\hline CEY_31 & 1.49 & .882 & 2.477 & 7.522 & CEY_69 & 2.43 & 1.449 & .880 & -.104 \\
\hline CEY_32 & 2.02 & 1.309 & 1.403 & 1.313 & CEY_70 & 2.42 & 1.427 & .847 & -.114 \\
\hline CEY_33 & 1.48 & .936 & 2.572 & 7.344 & CEY_71 & 2.18 & 1.252 & 1.075 & .564 \\
\hline CEY_34 & 1.44 & .815 & 2.449 & 7.369 & CEY_72 & 2.05 & 1.219 & 1.276 & 1.178 \\
\hline CEY_35 & 1.59 & 1.007 & 2.245 & 5.406 & CEY_73 & 2.19 & 1.237 & 1.139 & 1.041 \\
\hline CEY_36 & 1.75 & 1.125 & 1.912 & 3.879 & CEY_74 & 2.25 & 1.321 & .907 & .028 \\
\hline CEY_37 & 2.00 & 1.305 & 1.303 & .942 & CEY_75 & 2.35 & 1.419 & .871 & -.207 \\
\hline CEY_38 & 2.05 & 1.191 & 1.096 & .700 & & & & & \\
\hline
\end{tabular}

Nota: $M=$ media; $D E=$ desviación estándar; $g^{1}=$ asimetría; $g^{2}=$ curtósis. 
de mí si realmente me conociera», 23 «Soy indigno del amor, atención y respeto de otros», 24 «Siento que no soy simpático», 25 «Soy inaceptable de muchas fundamentales maneras como para revelarme auténticamente a otra gente», 33 «No puedo confiar en mi propio juicio para enfrentar las situaciones diarias» y 47 «Siento que no tengo otra elección que aceptar los deseos de otra gente, o de lo contrario se desquitará o me rechazará de alguna manera» (1.23 a 1.50).

\section{Análisis factorial exploratorio}

El análisis previo a la aplicación del AFE indicó una matriz de la determinante de .018. La medida de adecuación muestral de Kaiser-MeyerOlkin (KMO) obtuvo un valor de .927 (IC 95\% .918 a .919) considerado como muy bueno (Ferrando \& Anguiano-Carrasco, 2010; Hair, Anderson, Tatham, \& Black, 1999; Ruiz, Pardo, \& San Martín, 2010). Adicionalmente, la prueba de Bartlett también presentó un resultado adecuado para el cuestionario, $\chi^{2}$ $(45)=19072.3, p<.0001$ indicando suficiente intercorrelación de los ítems, al ser coherente la aplicación del análisis factorial en los datos de la muestra (Tabla 2).

Tabla 2

Matriz de correlaciones Pearson del cuestionario (YSQ-SF)

\begin{tabular}{ccccccccc}
\hline & F1 & F2 & F3 & F4 & F5 & F6 & F7 & F8 \\
\hline F1 & 1 & & & & & & & \\
F2 & .551 & 1 & & & & & & \\
F3 & .467 & .512 & 1 & & & & & \\
F4 & .460 & .423 & .705 & 1 & & & & \\
F5 & .540 & .504 & .645 & .535 & 1 & & & \\
F6 & .483 & .447 & .571 & .579 & .728 & 1 & & \\
F7 & .286 & .432 & .479 & .445 & .316 & .376 & 1 & \\
F8 & .426 & .533 & .544 & .534 & .562 & .537 & .389 & 1 \\
\hline
\end{tabular}

Para extraer y determinar el número de factores más apropiado se utilizó el criterio de análisis paralelo (Ferrando \& Lorenzo-Seva, 2014; Timmerman \& Lorenzo-Seva, 2011) y el criterio de Kaiser-Guttman o raíz latente (Ferrando \& Lorenzo-Seva, 2014; Hair et al., 1999). En ambos casos se coincide en una solución de ocho factores (ver Figura 1). El valor de la varianza explicada es 


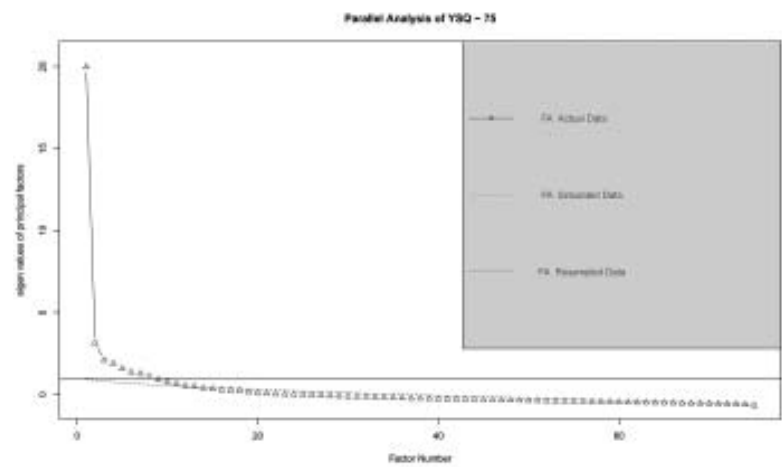

Figura 1. Análisis paralelo optimizado para la extracción de factores.

de $56.5 \%$, lo que supera el mínimo requerido de $40 \%$ (Lloret-Segura, FerreresTraver, Hernández-Baeza, \& Tomás-Marco, 2014).

\section{Análisis factorial confirmatorio}

La compleja estructura del YSQ-SF ha sido demostrada en estudios previos, por ello, se procedió a realizar un AFC basado en el modelo exploratorio de una solución de ocho factores con índices de ajuste parcialmente adecuados $x^{2}=2302.73$; CFI $=.89$, SRMR $=.058$; TLI $=.88$; RMSEA $=.045$ (IC 95\% .043 a .053) (ver Figura 2).

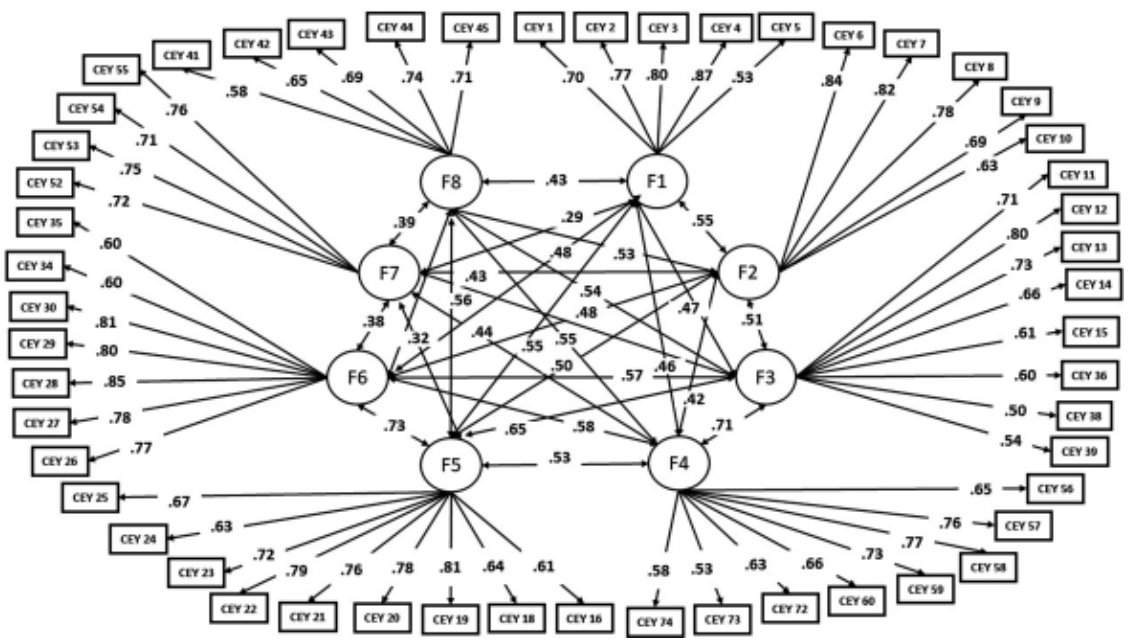

Figura 2. Análisis factorial confirmatorio del modelo de ocho factores para el YSQ-SF. 


\section{Fiabilidad de las puntuaciones}

La consistencia interna-homogeneidad mediante el índice de alfa fue para cada esquema adecuada. Sin embargo, la confiabilidad mínima se encontró en el esquema de abandono $(\mathrm{AB})$ con un valor de $\alpha=.70$, mientras que las puntuaciones de confiabilidad más alta fueron para el esquema de fracaso (FR) $\alpha=.92$. En cuanto a dimensiones, la confiabilidad mínima se encontró en la dimensión límites deteriorados con un valor de $\alpha=.82$.

\section{Tabla 3}

Confiabilidad del Cuestionario de Esquemas de Young YSQ-SF por dimensiones

\begin{tabular}{ll}
\hline \multicolumn{1}{c}{ Dimensiones } & $\alpha$ \\
\hline Desconexión/Rechazo & .92 \\
Autonomía Deteriorada & .91 \\
Limites Deteriorados & .82 \\
Orientación hacia los demás & .80 \\
Vigilancia Excesiva & .83 \\
\hline
\end{tabular}

\section{Discusión}

Debido al creciente aumento de problemas asociados a los esquemas disfuncionales tempranos, sobre todo en muestras no clínicas, como lo son los estudiantes universitarios, es importante que los profesionales de la salud mental cuenten con un instrumento adecuado y preciso que contribuya a la evaluación oportuna de estas conductas no adaptativas. No obstante, ser una escala de uso habitual en el contexto clínico-terapéutico de otros contextos diferentes al del Perú, en el presente estudio se ofrece un propuesta, aún inicial sobre la dimensionalidad de esta escala. Teniendo como objetivos analizar la estructura interna del instrumento y la fiabilidad de las puntuaciones en estudiantes peruanos.

A nivel de estructura interna no se pudo replicar la estructura de 15 factores como la propuesta original (Young, 2003). Esto, se debe básicamente a la complejidad del modelo en el contexto peruano con las particularidades de los estudiantes universitarios en los cuales predominan cierto grupo de 
esquemas; además del solapamiento de las dimensiones en este tipo de población específica.

La fiabilidad de las puntuaciones del cuestionario YSQ-SF para las dimensiones agrupadas fue adecuada bajo la evaluación de los supuestos sobre el coeficiente alfa, incluso los errores de medida aleatorios son similares, superando el estándar $(\alpha>.70)$, estos resultados corroboran el uso adecuado del YSQ-SF para la investigación. A pesar de ello, en este estudio no pudo evidenciarse un rango de precisión para contextos de evaluación clínica donde es requerido un $\alpha>.90$ (Mayo, 2015).

A pesar de que los resultados evidencian un apoyo a nivel aún exploratorio psicométrico para la versión en español del YSQ-SF, hay algunas limitaciones como la dificultad para emplear una medida de equivalencia entre otros contextos hispanohablantes (estudios interculturales), mayores rangos de edad y condición laboral, así como, la evaluación de puntos de corte. Dentro de esta línea, los posteriores trabajos deben estar encaminados hacia la evaluación de versiones complementarias del YSQ-SF con muestras de mayor diversidad (parejas, trabajadores, escolares y nivel cultural), que serían sustancialmente útiles tanto para la investigación como en el campo clínico.

\section{Referencias}

Ato, M., López, J., \& Benavente, A. (2013). Un sistema de clasificación de los diseños de investigación en psicología. Anales en psicología, 29(3), 1038-1059. doi: 10.6018/analesps.29.3.178511

Baranoff, J., Oei, T. P., Cho, S. H., \& Kwon, S. M. (2006). Factor structure and internal consistency of the Young Schema Questionnaire (Short Form) in Korean and Australian samples. Journal of Affective Disorders, 93(13), 133-140.

Caro, I. (2011). Hacia una práctica eficaz de las Psicoterapias Cognitivas. Bilbao: Descleé de Brouwer.

Cronbach, L. J. (1951). Coefficient alpha and the internal structure of tests. Psychometrika, 16(3), 297-334.

Ferrando, P. J., \& Anguiano-Carrasco, C. (2010). El análisis factorial como técnica de investigación en psicología. Papeles del Psicólogo, 31(1), 18-33.

Ferrando, P. J., \& Lorenzo-Seva, U. (2014). El análisis factorial exploratorio de los ítems: algunas consideraciones adicionales. Anales de psicología, 30(3), 1170-1175.

Graham, J. M. (2006). Congeneric and (essentially) tau-equivalent estimates of score reliability: what they are and how to use them.

Hair, J. F., Anderson, R. E., Tatham, R. L., \& Black, W. (1999). Análisis Multivariante. Madrid: Prentice Hall. 
Holgado-Tello, F. P., Chacón-Moscoso, S., Barbero-García, I., \& Vila-Abad, E. (2010). Polychoric versus Pearson correlations in exploratory and confirmatory factor analysis of ordinal variables. Quality \& Quantity, 44(1), 153-163

Hu, L., \& Bentler, P. (1998). Fit indices in covariance structure modeling: Sensivity to underparameterized model misspecification. Psychological Methods, 3(4), 424-453.

Kriston, L., Schäfer, J., von Wolff, A., Härter, M., \& Hölzel, L. P. (2012). The latent factor structure of Young's early maladaptive schemas: Are schemas organized into domains?. Journal of Clinical Psychology, 68(6), 684698.

Lachenal-Chevallet, K., Mauchand, P., Cottraux, J., Bouvard, M., \& Martin, R. (2006). Factor analysis of the schema questionnaire-short form in a nonclinical sample. Journal of Cognitive Psychotherapy, 20(3), 311.

Lee, S. Y., Poon, W. Y., \& Bentler, P. M. (1995). A two stage estimation of structural equation models with continuous and polytomous variables. British Journal of Mathematical and Statistical Psychology, 48(2), 339-358.

Lloret-Segura, S., Ferreres-Traver, A., Hernández-Baeza, A., \& Tomás-Marco, I. (2014). El Análisis Factorial Exploratorio de los Ítems: una guía práctica, revisada y actualizada. Anales de psicología, 30(3), 11511169. dx.doi.org/10.6018/analesps.30.3.199361

Lomax, R. G., \& Schumacker, R. E. (2017). A beginner's guide to structural equation modeling. psychology press.

Londoño, N., Schnitter, M., Marín, C., Calvete, E., Ferrer, A., Maestre, K. et al. (2012). Young Schema Questionnaire - Short Form: validación en Colombia. Universitas Psychologica, 11(1), 147-164.

Mauchand, P., Lachenal-Chevallet, K., \& Cottraux, J. (2011). Validation empirique du questionnaire abrégé des schémas de Young (YSQ-S2) sur une population de patients présentant un trouble de la personnalité borderline et de sujets témoins. L'Encéphale, 37(2), 138-143.

Mayo, A. M. (2015). Psychometric instrumentation: reliability and validity of instruments used for clinical practice, evidence-based practice projects and research studies. Clinical nurse specialist, 29(3), 134-138. doi: 10.1097/NUR.0000000000000131

Montero, I., \& León, O. (2002). Clasificación y descripción de las metodologías de investigación en Psicología. International Journal of Clinical and Health Psychology, 2(3), 503-508.

Montero, I., \& León, O. (2005). Sistema de clasificación del método en los informes de investigación en Psicología. International Journal of Clinical and Health Psychology, 5(1), 115-127.

Ruiz, M. A., Pardo, A., \& San Martín, R. (2010). Modelos de ecuaciones estructurales. Papeles del psicólogo, 31(1), 34-45.

Timmerman, M. E., \& Lorenzo-Seva, U. (2011). Dimensionality assessment of ordered polytomous items with parallel analysis. Psychological methods, 16(2), 209.

Young, J. (2003). Terapia cognitiva para transtornos da personalidade: Uma abordagem focada em esquemas. Porto Alegre: Artmed.

Young, J., \& Klosko, J. (2001). Reinventa tu vida. Cómo superar las actitudes negativas y sentirse bien de nuevo. Barcelona: Paidós.

Young, J., \& Klosko, J. (2007). Terapia de esquemas. En J. M. Oldham, A. E. Skodol y D. S. Bender (Eds.), Tratado de los trastornos de la personalidad (pp. 289-308). Barcelona: Elsevier Masson.

Young, J. E., \& Brown, G. (1994). Young schema questionnaire. Cognitive therapy for personality disorders: A schema-focused approach, 63-76.

Young, J., Klosko, J., \& Weishaar, M. (2013). Terapia de esquemas. Guía práctica. Bilbao: Descleé de Brouwer. 